\title{
Firehose instabilities triggered by the solar wind suprathermal electrons
}

\author{
S. M. Shaaban, ${ }^{1,2,3 \star}$ M. Lazar, ${ }^{1,2}$ R. A. López ${ }^{1}$ H. Fichtner, ${ }^{2}$ S. Poedts ${ }^{1}$ \\ ${ }^{1}$ Centre for Mathematical Plasma Astrophysics, KU Leuven, Celestijnenlaan 200B, B-3001 Leuven, Belgium. \\ ${ }^{2}$ Institut für Theoretische Physik, Lehrstuhl IV: Weltraum- und Astrophysik, Ruhr-Universität Bochum, D-44780 Bochum, Germany. \\ ${ }^{3}$ Theoretical Physics Research Group, Physics Department, Faculty of Science, Mansoura University, 35516, Mansoura, Egypt.
}

Accepted XXX. Received YYY; in original form ZZZ

\begin{abstract}
In collision-poor plasmas from space, e.g., solar wind, terrestrial magnetospheres, kinetic instabilities are expected to play a major role in constraining the temperature anisotropy of plasma particles, but a definitive answer can be given only after ascertaining their properties in these environments. The present study describes the full spectrum of electron firehose instabilities in the presence of suprathermal electron populations which are ubiquitous in space plasmas. Suprathermal electrons stimulate both the periodic and aperiodic branches, remarkable being the effects shown by the aperiodic mode propagating obliquely to the ambient magnetic field which markedly exceeds the growth rates of the parallel (periodic) branch reported recently in Lazar et al. (2017a, MNRAS 464, 564). Derived exclusively in terms of the plasma parameters, the anisotropy thresholds of this instability are also lowered in the presence of suprathermal electrons, predicting an enhanced effectiveness in the solar wind conditions. These results may also be relevant in various other astrophysical contexts where the firehose instabilities involve, e.g., solar flares, sites of magnetic field reconnection, accretion flows or plasma jets leading to shocks and co-rotating interactions in the heliosphere, interstellar medium, and galaxy clusters.
\end{abstract}

Key words: instabilities - solar wind - methods: numerical - waves - plasmas

\section{INTRODUCTION}

Preferential acceleration of charged particles along a guiding magnetic field is a common feature of any collision-poor plasmas expanding in our Universe, such as stellar winds, or plasma jets and accretion outflows (McComas et al. 2007; Paesold \& Benz 1999; Drake et al. 2006; Guo et al. 2014). An important amount of free energy is therefore expected to accumulate in the magnetic field direction, leading to kinetic anisotropies of plasma particles, like temperature $\left(T\right.$, or pressure $\left.P=n k_{B} T\right)$ anisotropies, e.g., $T_{\|}>T_{\perp}$, where $\|$ and $\perp$ denote directions with respect to the magnetic field. In the heliosphere, plasma is sufficiently dilute and kinetic anisotropies are easily triggered by the huge amount of energy released by the Sun via the more or less energetic outflows of charged particles. However, the observations unveil quasi-stable states with only small deviations from isotropy. Particle-particle collisions are inefficient at large heliocentric distances in the solar wind, and any excess of temperature (or mean kinetic energy) in a direction parallel to the magnetic field $\left(T_{\|}>T_{\perp}\right)$ is expected to be constrained by the self-generated instabilities. Of these, firehose instabilities appear to be the most plausible candidates, and if driven by the anisotropic electrons with an idealized bi-Maxwellian distribution, the theory

^ E-mail: shaaban.mohammed@kuleuven.be predicts two highly contrasting branches of electron firehose instability (EFHI) (Li \& Habbal 2000; Gary \& Nishimura 2003; Camporeale \& Burgess 2008; Hellinger et al. 2014): The periodic electron firehose (P-EFH) with a finite oscillation in time, i.e., $\mathfrak{R}(\omega) \neq 0$, also known as the non-resonant firehose branch (Gary \& Nishimura 2003), and the aperiodic electron firehose (A-EFH) with $\mathfrak{R}(\omega)=0$, which propagates only obliquely to the ambient magnetic field (i.e., $k_{\perp}>k_{\|}$, in terms of wave-vector components). In this case, the AEFH develops faster, with maximum growth rates much higher than P-EFH (Li \& Habbal 2000; Gary \& Nishimura 2003; Camporeale \& Burgess 2008; Hellinger et al. 2014), and may play the main role in reducing, eventually, the free energy, and leading to enhanced fluctuations which may scatter the electrons and limit their anisotropy. It is also known that firehose instability may influence macroscopic plasma properties, like viscous heating and thermal conduction, with implications for plasma dynamics at the magnetic field reconnection sites in the heliosheath (Schoeffler et al. 2011), and at larger scales in intracluster medium and accretion disks plasmas (Sharma et al. 2006), and may cause disruptions in the large-scale plasma jets triggering radiative fields (Subramanian et al. 2012).

For conditions more typical to the solar wind, the observed distributions show deviations from a standard Maxwellian shape, especially due to suprathermal populations, which enhance the highenergy tails and are well described by the (bi-)Kappa distribution 
functions (Pierrard \& Lazar 2010). One should thus expect that suprathermal electrons may contribute with an additional free energy, enhancing the unstable emissions. Such an expectation has recently been confirmed by a preliminary study which shows that growth rates of electron firehose instability propagating parallel to the magnetic field $\left(k=k_{\|}\right)$increase in the presence of suprathermal electrons (Lazar et al. 2017a). Here we characterize the full spectrum of firehose unstable modes under the influence of these suprathermal populations, obviously, with a focus on the oblique propagation, where both branches of the P-EFH and A-EFH instabilities are present.

The dispersion formalism is briefly described in the next section, on the basis of the general dispersion tensor for a bi-Kappa distributed plasma, which is given explicitly in the Appendix. The unstable firehose solutions are derived using an instability dispersion solver, named DSHARK and dedicated to plasmas with bi-Kappa components (Astfalk et al. 2015; Astfalk \& Jenko 2016). The effects of suprathermal electrons are outlined by contrasting with idealized solutions for bi-Maxwellian electrons. Choosing plasma parameters in the range of measurements in the solar wind enables us to compare with similar works (Gary \& Nishimura 2003; Camporeale \& Burgess 2008), which do not take into account the effects of suprathermal electrons. Sections 2.1 and 2.2 describe in detail both branches of firehose instabilities, for a case study (parametrized in Table 1) which is representative of the effects of suprathermal electrons. The fastest growing branch is also identified providing general instability conditions, exclusively, in terms of plasma parameters. Conclusions of the present study are summarized in section 3 .

\section{FULL SPECTRUM OF EFH INSTABILITIES}

The general linear dispersion relation for the electromagnetic modes propagating at an arbitrary angle $\theta$ with respect to the uniform magnetic field $\left(\boldsymbol{B}_{0}=B_{0} \hat{e}_{z}\right)$ in a bi-Kappa distributed plasma is given by (Summers et al. 1994; Shaaban et al. 2018a)

$$
0=\operatorname{det} D(\omega, k, \theta) \text {, }
$$

where $D(\omega, k, \theta)$ is a $3 \times 3$ matrix, whose elements are functions of wave-frequency $\omega$, wave-number $k$, and angle $\theta$, thermal velocity components, and the power-index $\kappa$. Explicit definitions of these elements are given in Appendix A. In a working frame co-moving with the solar wind the velocity distributions of the suprathermal electrons are described by the bi-Kappa distribution function

$f_{e}=\frac{\pi^{-3 / 2}}{\Theta_{e, \perp}^{2} \Theta_{e, \|}} \frac{\Gamma(\kappa+1)}{\kappa^{3 / 2} \Gamma(\kappa-1 / 2)}\left(1+\frac{v_{\|}^{2}}{\kappa \Theta_{e, \|}^{2}}+\frac{v_{\perp}^{2}}{\kappa \Theta_{e, \perp}^{2}}\right)^{-\kappa-1}$.

where $\int d^{3} v f_{e}=1, \kappa>3 / 2$ is the power-index, and $\Theta_{e, \|, \perp}$ are defined by the components of (kinetic) temperature (Lazar et al. 2017a)

$T_{e, \|}^{\kappa}=\frac{2 \kappa}{2 \kappa-3} \frac{m_{e}}{2 k_{B}} \Theta_{e, \|}^{2}$ and $T_{e, \perp}^{\kappa}=\frac{2 \kappa}{2 \kappa-3} \frac{m_{e}}{2 k_{B}} \Theta_{e, \perp}^{2}$,

assumed anisotropic, i.e., $T_{e, \|}^{\kappa}>T_{e, \perp}^{\kappa}$, with respect to the background magnetic field $\boldsymbol{B}_{0}$. The effects of protons are minimized by considering them isotropic and Maxwellian

$f_{p}(v)=\frac{1}{\pi^{3 / 2} \Theta_{p}^{3}} \exp \left(-\frac{v^{2}}{\Theta_{p}^{2}}\right)$,
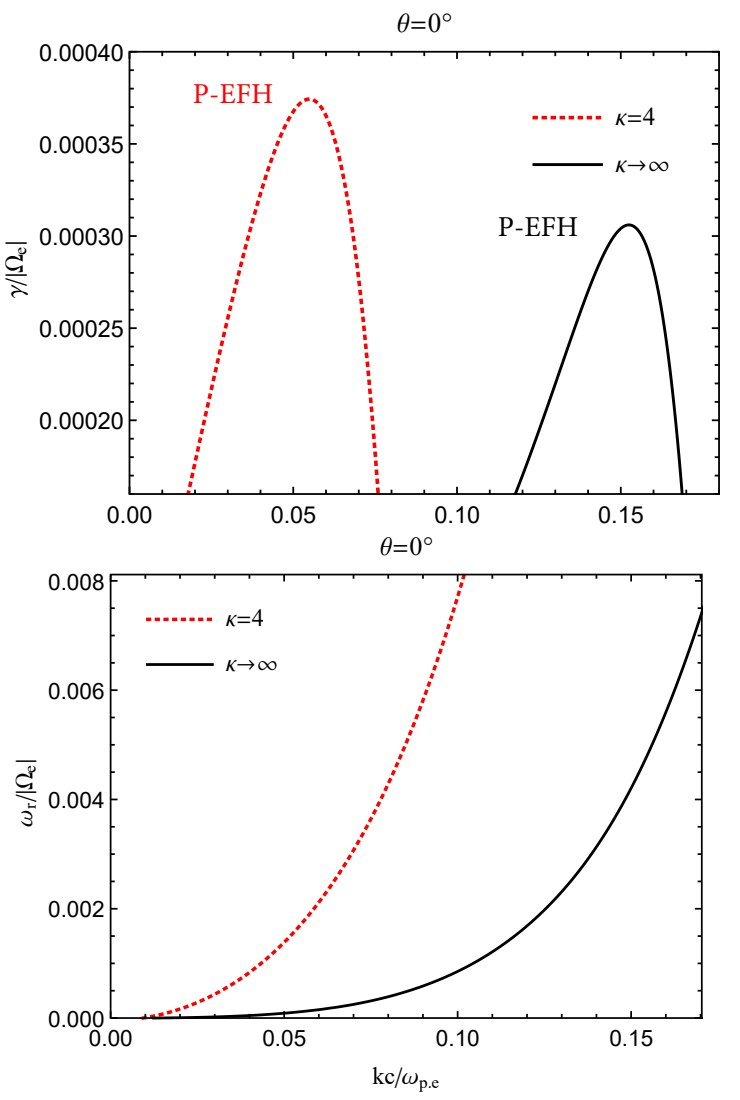

Figure 1. Growth rates (top) and wave frequencies (bottom) of the P-EFH instability at $\theta=0^{\circ}$ and different electron power-index $\kappa=4$ (red dotted lines), $\kappa \rightarrow \infty$ (black solid lines). Growth rates and wave frequencies are normalized to the electron parameters.

Table 1. Plasma parameters set used in the present study

\begin{tabular}{lcc}
\hline & Electrons $(i=e)$ & Protons $(i=p)$ \\
\hline$\beta_{i, \|}$ & 4 & 4 \\
$\beta_{i, \perp}$ & 2 & 4 \\
$A_{i}=T_{i, \perp} / T_{i, \|}$ & 0.5 & 1 \\
$m_{p} / m_{i}$ & 1836 & 1.0 \\
$\kappa$ & $4, \infty$ & $\infty$ \\
\hline
\end{tabular}

where $\Theta_{p}=\sqrt{2 k_{B} T_{p} / m_{p}}$ is the proton thermal velocity.

Numerical analysis of the firehose unstable solutions is performed using DSHARK solver (Astfalk et al. 2015), recently developed to resolve the dispersion relation (1) for modes propagating at arbitrary angles with respect to the background magnetic field. Plasma parameters are tabulated in Table 1, unless elsewhere provided. The effects of suprathermal electrons are outlined by contrasting the results obtained for a power-index $\kappa=4$ with those for a bi-Maxwellian limit $(\kappa \rightarrow \infty)$ approaching the thermal core population of lower temperatures (Lazar et al. 2017a)

$T_{\|, \perp}<T_{\|, \perp}^{\kappa}=\frac{2 \kappa}{2 \kappa-3} T_{\|, \perp}$, or $\beta_{\|, \perp}<\beta_{\|, \perp}^{\kappa}=\frac{2 \kappa}{2 \kappa-3} \beta_{\|, \perp}$,

where the plasma beta parameter $\beta \equiv 8 \pi n_{e} k_{B} T / B_{0}^{2}$. 
(a) $\theta=15^{\circ}$

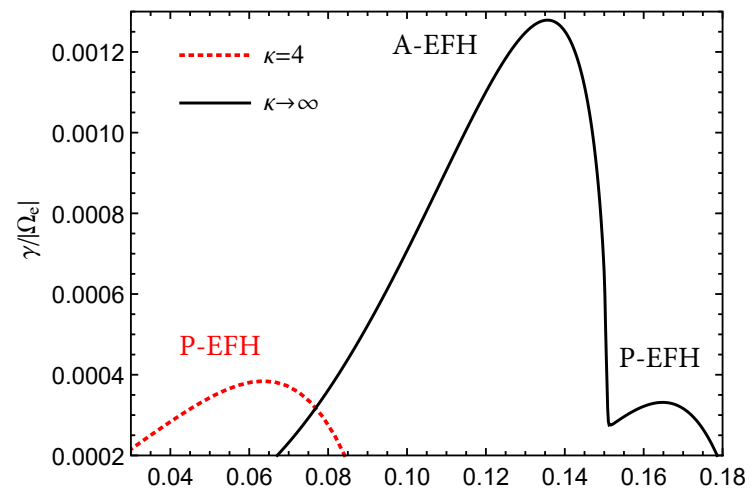

(b) $\theta=26^{\circ}$

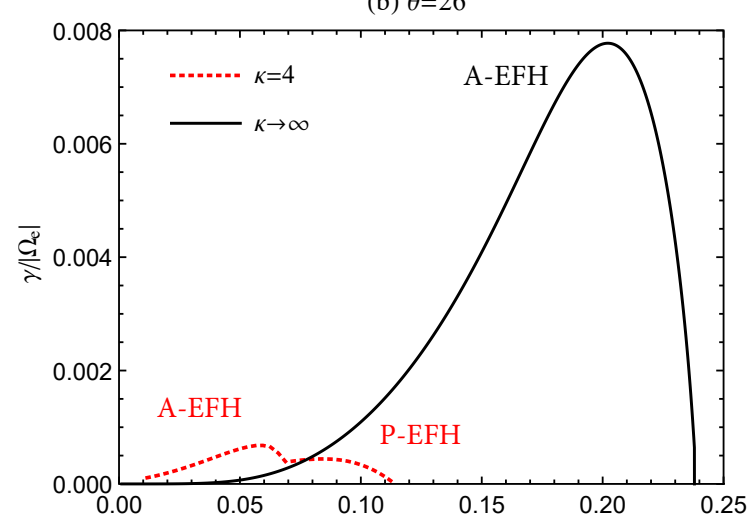

(c) $\theta=54^{\circ}$

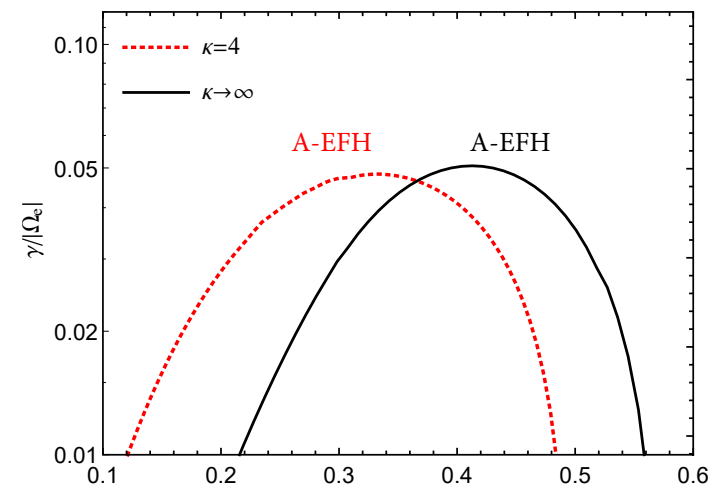

(d) $\theta=70^{\circ}$

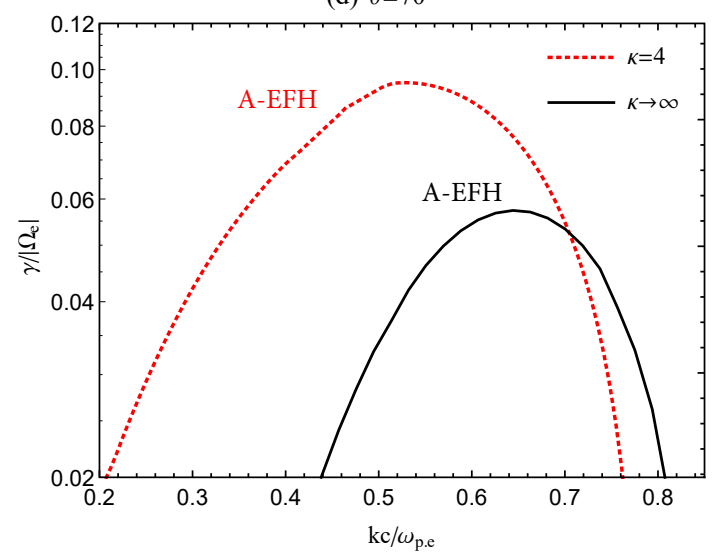

Figure 2. Growth rates of the EFH instabilities for various angles of propagation $\theta$ and different electron power-index $\kappa=4$ (red dotted lines), $\kappa \rightarrow \infty$ (black solid lines).

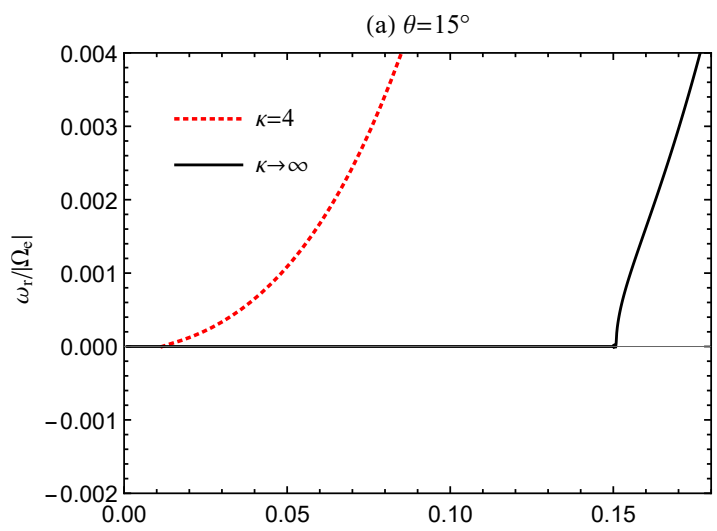

(b) $\theta=26^{\circ}$

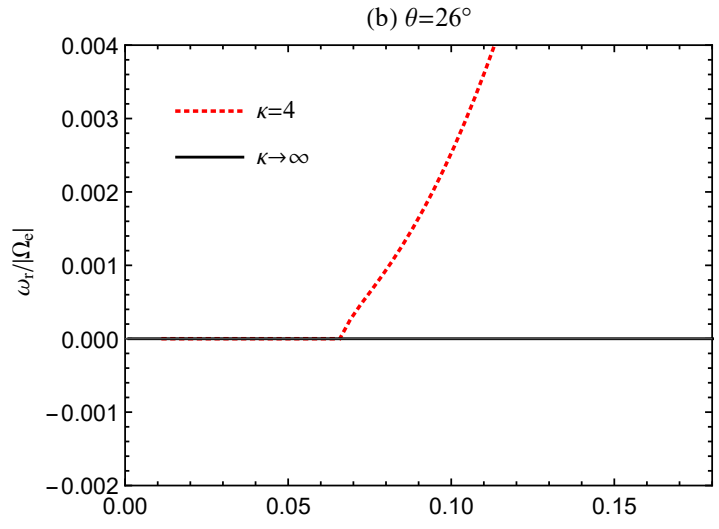

(c) $\theta=54^{\circ}$

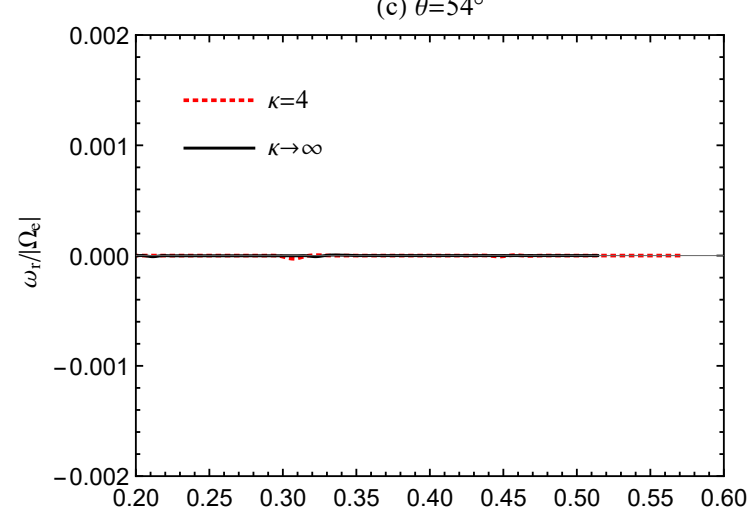

(d) $\theta=70^{\circ}$

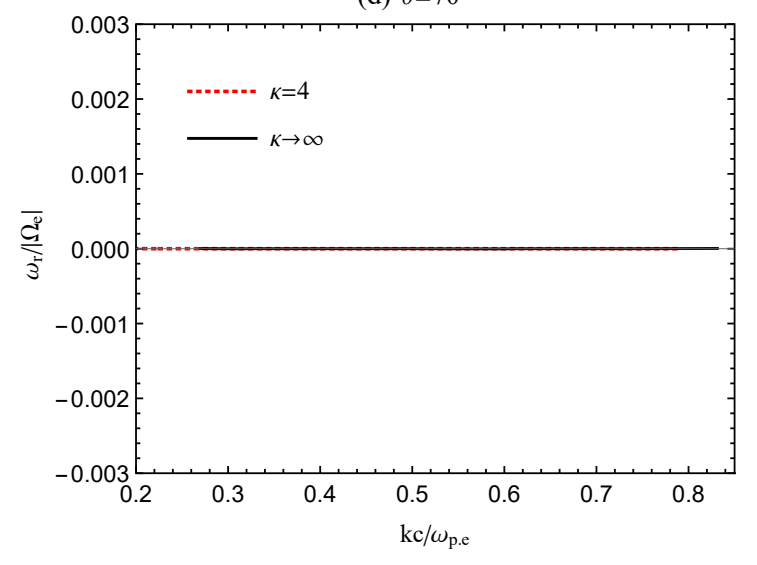

Figure 3. The corresponding wave frequencies of the EFH instabilities growth rates in Figure 2. 


\subsection{Numerical solutions}

In order to build a comprehensive picture for the effects of suprathermal electrons we start with a comparative analysis of P-EFH and A-EFH branches, e.g., in Figures 1-3, for the plasma conditions in Table 1 and various angles of propagation. The test case in Figure $1\left(A_{e}=0.5\right.$ and $\left.\beta_{e}, \|=4\right)$ presents the dispersive and stability properties of the P-EFH instability in direction parallel to the magnetic field, i.e., $\theta=0^{\circ}$, and confirms the validity of a new normalization introduced in DSHARK with respect to the electron (scaling) parameters (replacing the original normalization to ion parameters). Both the maximum growth-rate and wave-frequency of the P-EFH instability increase in the presence of suprathermal electrons (red-dotted lines), i.e. for finite (low) values of $\kappa$, and the instability becomes more operative at lower wave-numbers. These results obtained with DSHARK are in perfect agreement with the P-EFH solutions provided by Lazar et al. (2017a).

The unstable solutions in Figures 2 and 3, displaying, respectively, the growth rates and the corresponding wave frequencies, enable us to examine the effect of suprathermal electrons on the oblique modes, i.e., for $\theta=15^{\circ}, 26^{\circ}, 54^{\circ}$, and $70^{\circ}$. These angles of propagation are carefully chosen to highlight the most important effects of suprathermals on the EFH instabilities. At small angles, i.e., $\theta=15^{\circ}, 26^{\circ}$, both P-EFH and A-EFH branches are present with modest growth-rates (Figure 2), but restrain to lower wave numbers in the presence of suprathermals (red-dotted lines). Stimulation of P-EFH instability by the suprathermals, reported by Lazar et al. (2017a) for parallel propagation $\left(\theta=0^{\circ}\right)$, is also observed for the oblique angles, e.g., in Figure 2, panel (a), where both P-EFH peaks are present. For $\theta=26^{\circ} \mathrm{P}$-EFH unstable modes are still present only in the presence of suprathermals (black-solid line), when growth rates in panel (b) display two distinct peaks of A-EFH and P-EFH at low and large wave-numbers, respectively. This branch disappears with increasing $\theta$, and the peak of A-EFH instability significantly increases for a highly oblique propagation, e.g., for $\theta>50^{\circ}$ in panels (c) and (d). The fastest growing modes, corresponding to the peaking growth rates at these angles, are markedly stimulated by the suprathermal electrons. The corresponding wave frequencies are displayed in Figure 3, enabling to identify and distinguish between different branches of EFH solutions. P-EFH unstable modes are LH polarized with $\mathfrak{R}(\omega)>0$, while A-EFH modes have zero real frequency $\mathfrak{R}(\omega)=0$. The wave frequency confirms the effect of suprathermal (energetic) electrons, in the presence of which the range of unstable wave-numbers translates to markedly lower values, for both instabilities, P-EFH at small angles, and A-EFH at highly oblique angles.

Maximum growth rates $\gamma_{m} / \Omega_{p}$ and the corresponding wavenumbers $k_{m} c / \omega_{p, p}$ are plotted in Figure 4, panels (a) and (b), respectively, as a function of $\theta$. P-EFH instability is indicated with solid circles, while open circles represent A-EFH instability. Standard Maxwellian $(\kappa \rightarrow \infty)$ solutions (black) show profiles similar to those obtained in Paesold \& Benz (1999), e.g., growth rates of A-EFH mode exceed the P-EFH mode at about $\theta \simeq 10^{\circ}$; a nonuniform variation of maximum growth rates which increase and then decrease with increasing angle of propagation, reaching the peak at about $65^{\circ}$ (also in agreement with the results in Maneva et al. (2016)). Solutions obtained under the influence of suprathermal electrons $(\kappa=4$, red) show that A-EFH branch becomes faster than the P-EFH at about $30^{\circ}$, and undergo a similar non-uniform variation with $\theta$, reaching its peak at about $72^{\circ}$. We can already point out two results: (i) for both P-EFH and A-EFH branches the peaks at oblique angles become more pronounced in the presence (a)

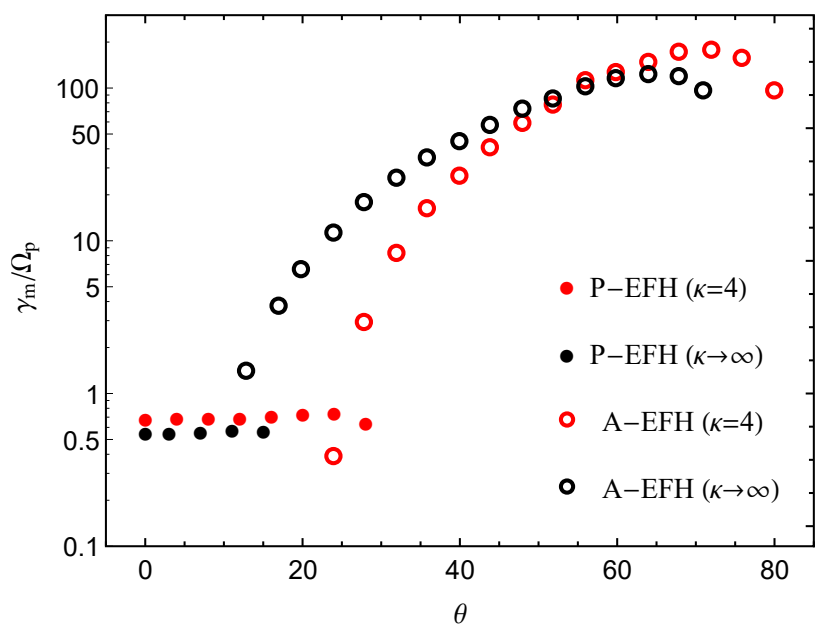

(b)

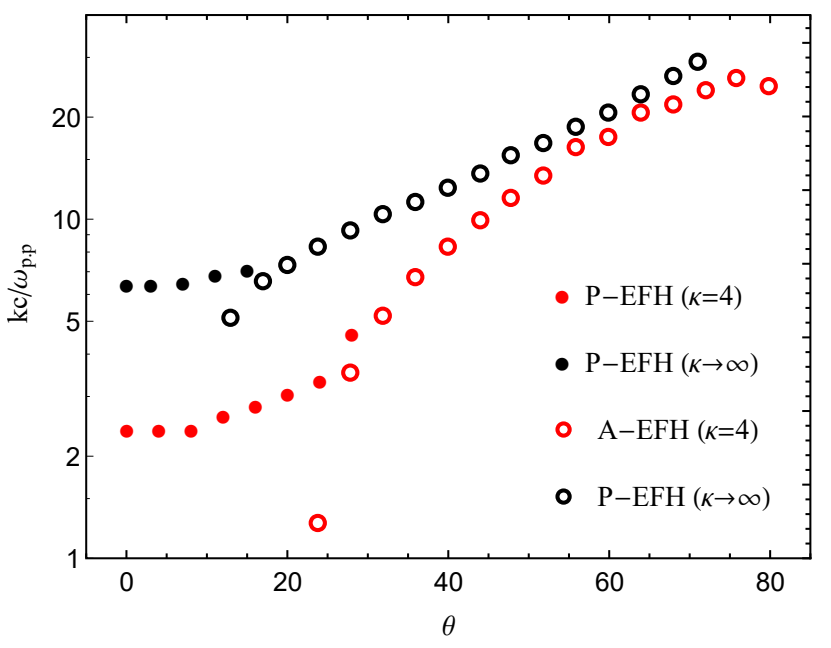

Figure 4. Maximum growth rates, panel (a), and the corresponding maximum wave-number, panel (b), of P-EFH (solid circles) and A-EFH (open circles), for bi-Kappa ( $\kappa=4$, red) and bi-Maxwellian distributed electrons $(\kappa \rightarrow \infty$, blue). We normalized to the proton parameters for a better visualization.

of suprathermals, and are obtained for slightly higher angles; (ii) A-EFH remains the fastest growing mode, and becomes even faster under the influence of suprathermals. The wave-numbers $k_{m} c / \omega_{p, p}$ corresponding to maximum growth rates in panel (b) increase with increasing $\theta$, and show that, whenever the growth rates display two peaks, i.e., for P-EFH and A-EFH branches, the peak of aperiodic mode is located at lower wave-numbers. Moreover, the effect of suprathermal electrons, lowering the unstable wave-numbers is again evident.

Of these two branches of EFH instabilities, the aperiodic branch (A-EFH) is by far the fastest growing and may therefore be the most effective in the relaxation of temperature anisotropy. Figure 5 provides a better visualization of its properties by mapping with contour plots the maximum growth rate as a function of the wave number $k c / \omega_{p, e}$ and the angle of propagation $\theta$. Panel (a) displays the solutions in the presence of suprathermal electrons $(\kappa=4)$ with maximum growth rates (color bar) peaking at $\gamma_{m} /\left|\Omega_{e}\right| \approx 0.095$ (for $\theta_{m}=72^{\circ}$ ), markedly higher than the peak of Maxwellian solutions at $\gamma_{m} /\left|\Omega_{e}\right| \approx 0.065$ (for $\theta_{m}=65^{\circ}$ ) in panel (b). For both cases peaks are obtained at $k_{m} \approx 0.55 \omega_{p, e} / c$. These results show clearly 


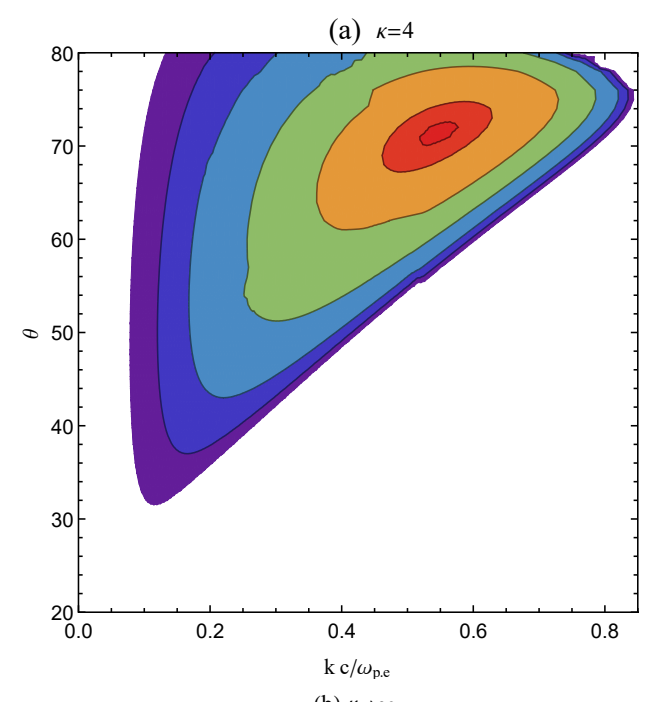

$\gamma /\left|\Omega_{\theta}\right|$

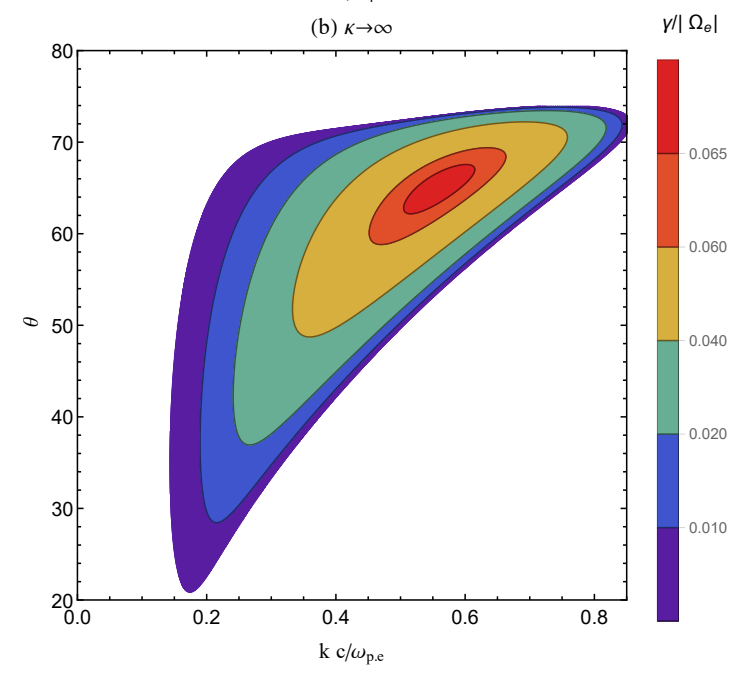

Figure 5. 3D representation of the A-EFH maximum growth rates as a function of $\theta$ and $k c / \omega_{p, e}$ for $\kappa=4$, panel (a) and $\kappa \rightarrow \infty$, panel (b).

that A-EFH instability is stimulated in the presence of suprathermal electrons, and the fastest growing mode develops at a higher angle of propagation.

\subsection{The influence of suprathermal electrons on A-EFH thresholds}

In this section we describe the anisotropy thresholds of the aperiodic branch identified as the fastest growing mode. These thresholds characterize the unstable plasma conditions for the lower levels of maximum growth rates, approaching marginal condition of stability $\left(\gamma_{m} /\left|\Omega_{e}\right| \rightarrow 0\right)$. Here we compare the anisotropy thresholds derived for a maximum growth rate $\gamma_{m}=10^{-2}\left|\Omega_{e}\right|$. Figure 6 displays these isocontours as a function of $\theta$ and complementary anisotropy $1-T_{\perp} / T_{\|}$for $\kappa=4$ (red) and Maxwellian limit $(\kappa \rightarrow \infty$, black). These thresholds show a non-monotonous variation, decreasing and then increasing with increasing $\theta$. In the presence of suprathermals $(\kappa=4)$ the lowest (minimum) anisotropy $\left(1-T_{\perp} / T_{\|}\right)_{m}=0.292$ is found at $\theta_{m} \simeq 56^{\circ}$ (opened circle on red line), and is much lower than that obtained for Maxwellian limit, i.e., $\left(1-T_{\perp} / T_{\|}\right)_{m}=0.36$ at $\theta_{m} \simeq 48.5^{\circ}$ (opened circle on black line). Evaluating the lowest (threshold) anisotropies for different values of electron (parallel)

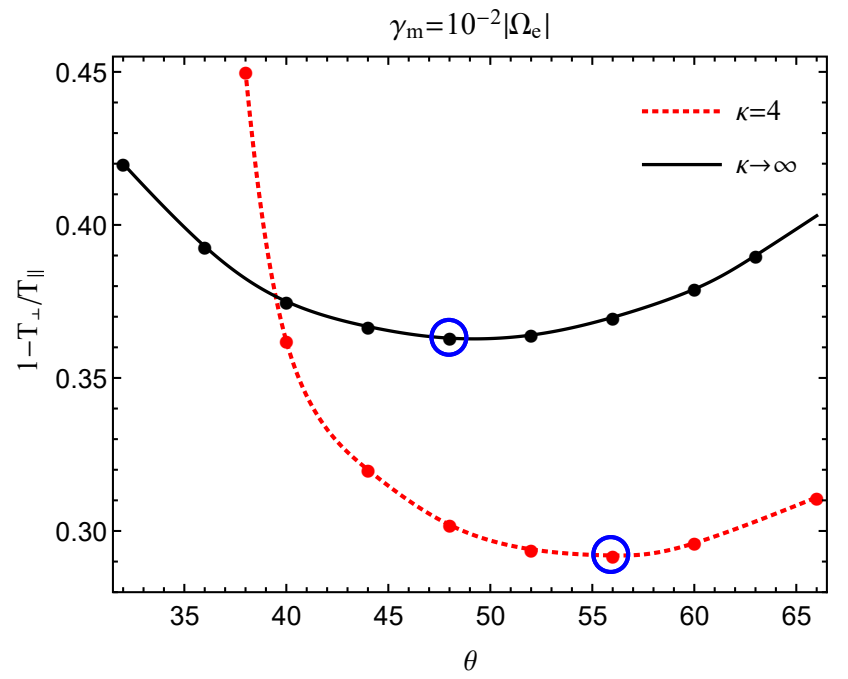

Figure 6. Complementary anisotropy $\left(1-T_{\perp} / T_{\|}\right)$thresholds for maximum growth rates $\gamma_{m}=10^{-2}\left|\Omega_{e}\right|$ of the A-EFH instability, as a function of $\theta$, for $\kappa=4$ (red dotted line) and $\kappa \rightarrow \infty$ (black solid line). Blue open circles indicate the angles $\theta_{m}$ corresponding to the lowest anisotropy.

plasma beta $\beta_{\|}=2,3,4,5,6,8,20,50$, enabled us to determine the general instability thresholds, as a function of plasma parameters, e.g., $\beta_{\|}$. These thresholds are displayed in Figure 7 using temperature anisotropy introduced in Table 1, i.e., $T_{\perp} / T_{\|}$(as also used in recent studies to facilitate comparison with the observations). As a function of $\beta_{\|}$, this threshold is fitted to an inverse correlation law (Gary \& Nishimura 2003)

$\frac{T_{\perp}}{T_{\|}}=1-\frac{a}{\beta_{\|}^{b}}$,

where for the fitting parameters we find $(a, b)=(0.96,0.86)$ in the presence of suprathermal electrons $(\kappa=4$, dotted-red), and $(a, b)=(1.27,0.90)$ for Maxwellian limit $(\kappa \rightarrow \infty$, solid-black). The instability thresholds are determined for an extended range of electron plasma beta $1<\beta_{\|}<70$, to include conditions specific to various plasma conditions in heliosphere, e.g., solar flares, solar wind or planetary magnetospheres (Štverák et al. 2008).

Suprathermal electrons have a stimulating effect on the instability, diminishing the threshold to lower values of $\beta_{\|}$and closer to isotropy $(A \rightarrow 1)$, as shown in Figure 7 . The unstable regime expands confirming results in Figures 5 and 6. This stimulation is indeed more pronounced at low values of $\beta_{\|}$, but decreases by increasing this parameter.

\section{CONCLUSIONS}

In-situ measurements of the solar wind electrons suggest that their velocity distributions exhibit power-law tails well described by the bi-Kappa functions (Vasyliunas 1968; Pierrard \& Lazar 2010; Štverák et al. 2008). Recent studies show that suprathermal electrons, if correctly interpreted, can significantly change the (linear) dispersive characteristics of kinetic instabilities (Viñas et al. 2015; Lazar et al. 2015; Shaaban et al. 2016; Lazar et al. 2017b; Shaaban et al. 2017, 2018b). However, these studies are in general restricted to modes propagating parallel to the background magnetic field, while the obliquely propagating unstable modes are rarely investigated and only for idealized (bi-)Maxwellian plasmas. In the present work, we 


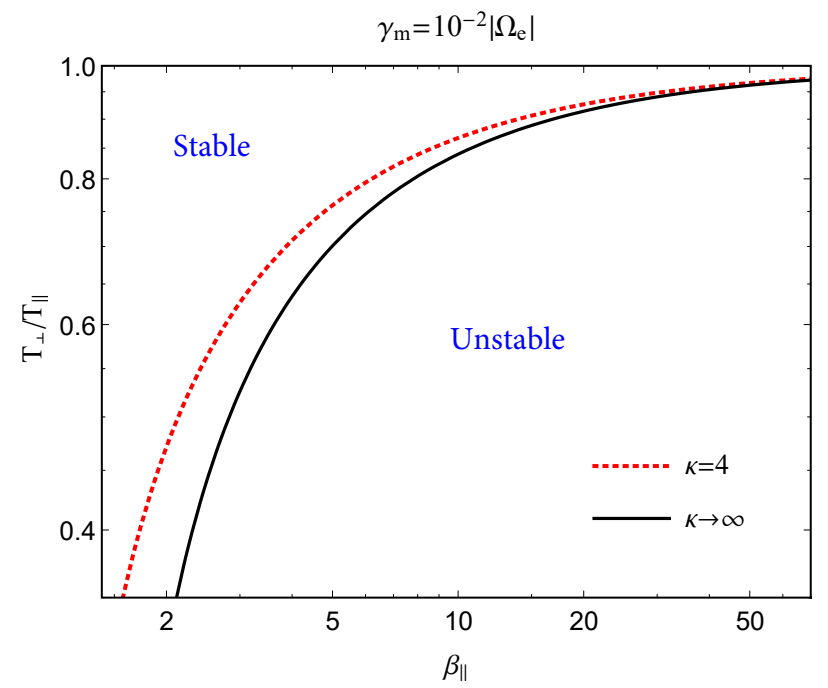

Figure 7. Electron anisotropy threshold (maximum growth rates $\gamma_{m}=$ $10^{-2}\left|\Omega_{e}\right|$ ) for A-EFH instability as a function of parallel plasma beta $\beta_{\|}$for $\kappa=4$ (red dotted line) and $\kappa \rightarrow \infty$ (black solid line).

have analyzed the full spectrum of firehose instabilities triggered by the anisotropic electrons in the presence of their suprathermal populations. The general linear dispersion relations have been solved for arbitrary angles of propagation using an advanced numerical solver dedicated to plasmas with bi-Kappa populations, named DSHARK (Astfalk et al. 2015).

In Section 2 we have described both the periodic and aperiodic branches of the electron firehose unstable solutions for a set of plasma parameters typically experienced in the solar wind. In the oblique directions, the periodic branch extends only to low angles, while the aperiodic firehose instability develops with much higher growth rates at highly oblique directions. After a detailed description in Figures 1-3, which teaches us how to differentiate between these two branches, in Figure 4 we have shown that suprathermal electrons stimulate both branches, increasing the growth rates of the fastest growing modes as indicated by the peaking (maximum) growth rates. The aperiodic firehose remains the fastest growing and Figure 5 provides a complete picture of the influence of suprathermal electrons on its peaking growth rates, as this depends on the wave-number and the propagation angle. The anisotropy thresholds in Figures 6 and 7 provide an important indication about conditions limiting the instability of aperiodic firehose mode. Particularly important are the general thresholds provided in Figure 7, which are derived exclusively in terms of plasma parameters, and show that conditions favorable to this instability may significantly extend in the presence of suprathermal electrons.

To conclude, we have shown that suprathermal electrons present in space plasmas, and in particular in the solar wind and planetary environments, have an important impact stimulating both branches of the firehose instability, of which the aperiodic firehose remains dominant, and may induce new regimes of instability at lower wave-numbers. Comparing to standard results for bi-Maxwellian plasmas, which ignore the effects of suprathermal electrons, our results predict an enhanced role of firehose instabilities, especially in collision-poor plasmas from space where these instabilities can prevent the large deviations from isotropy and, thus, explain the observations. These new regimes of firehose instability may also be relevant for many other scenarios in astrophysics which assume an implication of this instability, e.g., solar flares, sites of magnetic field reconnection, accretion flows or plasma jets leading to shocks and co-rotating interactions in the heliosphere, interstellar medium and galaxy clusters.

\section{ACKNOWLEDGEMENTS}

These results were obtained in the framework of the projects SCHL 201/35-1 (DFG-German Research Foundation), GOA/2015014 (KU Leuven), G0A2316N (FWO-Vlaanderen), and C 90347 (ESA Prodex 9). S.M. Shaaban acknowledges support by a FWO Postdoctoral Fellowship (Grant No. 12Z6218N), and a FWO Travel grant for long stay abroad (Grant No. V419818N). Thanks are due to Patrick Astfalk for valuable discussions and for providing the DSHARK code. We also appreciate the support from the International Space Science Institute (ISSI) for hosting the international ISSI team on Kappa Distributions, which triggered fruitful discussions that were beneficial for the work presented here.

\section{REFERENCES}

Astfalk P., Jenko F., 2016, J. Geophys. Res., 121, 2842

Astfalk P., Görler T., Jenko F., 2015, J. Geophys. Res., 120, 7107

Camporeale E., Burgess D., 2008, J. Geophys. Res., 113, A07107

Drake J. F., Swisdak M., Che H., Shay M. A., 2006, Nature, 443, 553

Gary S. P., Nishimura K., 2003, Physics of Plasmas, 10, 3571

Guo X., Sironi L., Narayan R., 2014, ApJ, 797, 47

Hellinger P., Trávníček P. M., Decyk V. K., Schriver D., 2014, J. Geophys. Res., 119, 59

Lazar M., Poedts S., Fichtner H., 2015, A\&A, 582, A124

Lazar M., Shaaban S. M., Poedts S., Štverák Š., 2017a, MNRAS, 464, 564

Lazar M., Pierrard V., Shaaban S. M., Fichtner H., Poedts S., 2017b, A\&A, 602, A44

Li X., Habbal S. R., 2000, J. Geophys. Res., 105, 27377

Maneva Y., Lazar M., Viñas A., Poedts S., 2016, ApJ, 832, 64

McComas D. J., et al., 2007, Rev. Geophys., 45, RG1004

Paesold G., Benz A. O., 1999, A\&A, 351, 741

Pierrard V., Lazar M., 2010, Sol. Phys., 267, 153

Schoeffler K. M., Drake J. F., Swisdak M., 2011, ApJ, 743, 70

Shaaban S. M., Lazar M., Poedts S., Elhanbaly A., 2016, Ap\&SS, 361, 193 Shaaban S. M., Lazar M., Poedts S., Elhanbaly A., 2017, Ap\&SS, 362, 13

Shaaban S. M., Lazar M., Astfalk P., Poedts S., 2018a, J. Geophys. Res., 123,1754

Shaaban S. M., Lazar M., Poedts S., 2018b, MNRAS, 480, 310

Sharma P., Hammett G. W., Quataert E., Stone J. M., 2006, ApJ, 637, 952

Štverák Š., Trávníček P., Maksimovic M., Marsch E., Fazakerley A. N., Scime E. E., 2008, J. Geophys. Res., 113, A03103

Subramanian P., Shukla A., Becker P. A., 2012, MNRAS, 423, 1707

Summers D., Xue S., Thorne R. M., 1994, Physics of plasmas, 1, 2012

Vasyliunas V. M., 1968, J. Geophys. Res., 73, 2839

Viñas A. F., Moya P. S., Navarro R. E., Valdivia J. A., Araneda J. A., Muñoz V., 2015, J. Geophys. Res., 120, 3307

\section{APPENDIX A: ELEMENTS OF THE DIELECTRIC TENSOR}

The general linear dispersion relations for the plasma electromagnetic modes propagating at an arbitrary angle with respect to the uniform background magnetic field, $\boldsymbol{B}_{0}=B_{0} \hat{e}_{z}$, in a bi-kappa distributed plasma is given by (Summers et al. 1994; Shaaban et al. 2018a)

$0=\operatorname{det} D(\omega, k, \theta)$, 
with

$D(\omega, k, \theta)=$

$$
\left|\begin{array}{ccc}
1-\frac{c^{2} k_{\|}^{2}}{w^{2}}+\epsilon_{x x} & \epsilon_{x y} & \frac{c^{2} k_{\|} k_{\perp}}{w^{2}}+\epsilon_{x z} \\
\epsilon_{y x} & 1-\frac{c^{2} k^{2}}{w^{2}}+\epsilon_{y y} & \epsilon_{y z} \\
\frac{c^{2} k_{\|} k_{\perp}}{w^{2}}+\epsilon_{z x} & \epsilon_{z y} & 1-\frac{c^{2} k_{\perp}^{2}}{w^{2}}+\epsilon_{z z}
\end{array}\right|
$$

The dielectric elements in Eq.(A2) can be expressed in terms of the modified plasma dispersion function $Z_{\kappa}\left(\xi_{j}\right)$ and the Bessel functions of the first kind $J_{n}(\mu)$ as follows

$$
\begin{aligned}
& \epsilon_{x x}=\sum_{j} \sum_{n=-\infty}^{n=+\infty} \frac{\omega_{p, j}^{2}}{\omega^{2}} \frac{n^{2}}{\lambda_{j}^{2}}\left[\Lambda_{j} K_{1} \int_{0}^{\infty} \frac{\mu J_{n}^{2}(\mu) d \mu}{\left(1+\mu^{2} / 2 \lambda \kappa\right)^{\kappa+3 / 2}}\right. \\
& +\left(\Lambda_{j} \xi_{j}+\frac{\omega}{k_{\|} \Theta_{\|, j}}\right) K_{2} \int_{0}^{\infty} \frac{\mu J_{n}^{2}(\mu) d \mu}{\left(1+\mu^{2} / 2 \lambda \kappa\right)^{\kappa+2}} \\
& \left.\times Z_{\kappa+1}\left(\xi_{j} \sqrt{\frac{\kappa+1}{1+\mu^{2} / 2 \lambda}}\right) d \mu\right] \\
& \epsilon_{y y}=\sum_{j} \sum_{n=-\infty}^{n=+\infty} \frac{\omega_{p, j}^{2}}{\omega^{2} \lambda_{j}^{2}}\left[\Lambda_{j} K_{1} \int_{0}^{\infty} \frac{\mu^{3} J_{n}^{\prime 2}(\mu) d \mu}{\left(1+\mu^{2} / 2 \lambda \kappa\right)^{\kappa+3 / 2}}\right. \\
& +\left(\Lambda_{j} \xi_{j}+\frac{\omega}{k_{\|} \Theta_{\|, j}}\right) K_{2} \int_{0}^{\infty} \frac{\mu^{3} J_{n}^{\prime 2}(\mu) d \mu}{\left(1+\mu^{2} / 2 \lambda \kappa\right)^{\kappa+2}} \\
& \left.\times Z_{\kappa+1}\left(\xi_{j} \sqrt{\frac{\kappa+1}{1+\mu^{2} / 2 \lambda}}\right) d \mu\right] \\
& \epsilon_{z z}=\sum_{j} \sum_{n=-\infty}^{n=+\infty} \frac{\omega_{p, j}^{2}}{\omega^{2}} \frac{\Theta_{\|, j}^{2}}{\Theta_{\perp, j}^{2}} \frac{2 \xi_{j}}{\lambda^{3 / 2}}\left(\Lambda_{j} \xi_{j}+\frac{\omega}{k_{\|} \Theta_{\|, j}}\right) \\
& \times\left[K_{1} \int_{0}^{\infty} \frac{\mu J_{n}^{2}(\mu) d \mu}{\left(1+\mu^{2} / 2 \lambda \kappa\right)^{\kappa+3 / 2}}+\xi_{j} K_{2} \int_{0}^{\infty} \frac{\mu J_{n}^{2}(\mu) d \mu}{\left(1+\mu^{2} / 2 \lambda \kappa\right)^{\kappa+2}}\right. \\
& \left.\times Z_{\kappa+1}\left(\xi_{j} \sqrt{\frac{\kappa+1}{1+\mu^{2} / 2 \lambda}}\right) d \mu\right] \\
& \epsilon_{x y}=-\epsilon_{y x}=\sum_{j} \sum_{n=-\infty}^{n=+\infty} \frac{\omega_{p, j}^{2}}{\omega^{2}} \frac{i n}{\lambda_{j}^{2}}\left[\Lambda_{j} K_{1} \int_{0}^{\infty} \frac{\mu^{2} J_{n}(\mu) J_{n}^{\prime}(\mu) d \mu}{\left(1+\mu^{2} / 2 \lambda \kappa\right)^{\kappa+3 / 2}}\right. \\
& +\left(\Lambda_{j} \xi_{j}+\frac{\omega}{k_{\|} \Theta_{\|, j}}\right) K_{2} \int_{0}^{\infty} \frac{\mu^{2} J_{n}(\mu) J_{n}^{\prime}(\mu) d \mu}{\left(1+\mu^{2} / 2 \lambda \kappa\right)^{\kappa+2}} \\
& \left.\times Z_{\kappa+1}\left(\xi_{j} \sqrt{\frac{\kappa+1}{1+\mu^{2} / 2 \lambda}}\right) d \mu\right] \\
& \epsilon_{x z}=\epsilon_{z x}=\sum_{j} \sum_{n=-\infty}^{n=+\infty} \frac{\omega_{p, j}^{2}}{\omega^{2}} \frac{\Theta_{\|, j}}{\Theta_{\perp, j}} \frac{\sqrt{2} n}{\lambda^{3 / 2}}\left(\Lambda_{j} \xi_{j}+\frac{\omega}{k_{\|} \Theta_{\|, j}}\right) \\
& \times\left[K_{1} \int_{0}^{\infty} \frac{\mu J_{n}^{2}(\mu) d \mu}{\left(1+\mu^{2} / 2 \lambda \kappa\right)^{\kappa+3 / 2}}+\xi_{j} K_{2} \int_{0}^{\infty} \frac{\mu J_{n}^{2}(\mu) d \mu}{\left(1+\mu^{2} / 2 \lambda \kappa\right)^{\kappa+2}}\right. \\
& \left.\times Z_{\kappa+1}\left(\xi_{j} \sqrt{\frac{\kappa+1}{1+\mu^{2} / 2 \lambda}}\right) d \mu\right]
\end{aligned}
$$

$$
\begin{aligned}
\epsilon_{y z} & =-\epsilon_{z y}=\sum_{j} \sum_{n=-\infty}^{n=+\infty} \frac{\omega_{p, j}^{2}}{\omega^{2}} \frac{\Theta_{\|, j}}{\Theta_{\perp, j}} \frac{-\sqrt{2} i}{\lambda^{3 / 2}}\left(\Lambda_{j} \xi_{j}+\frac{\omega}{k_{\|} \Theta_{\|, j}}\right) \\
& \times\left[K_{1} \int_{0}^{\infty} \frac{\mu^{2} J_{n}(\mu) J_{n}^{\prime}(\mu) d \mu}{\left(1+\mu^{2} / 2 \lambda \kappa\right)^{\kappa+3 / 2}}+\xi_{j} K_{2} \int_{0}^{\infty} \frac{\mu^{2} J_{n}(\mu) J_{n}^{\prime}(\mu) d \mu}{\left(1+\mu^{2} / 2 \lambda \kappa\right)^{\kappa+2}}\right. \\
& \left.\times Z_{\kappa+1}\left(\xi_{j} \sqrt{\frac{\kappa+1}{1+\mu^{2} / 2 \lambda}}\right) d \mu\right]
\end{aligned}
$$

where $j$ denotes different plasma species, e.g., electrons (subscript $j=e$ ), protons $(j=p), \omega_{p, j}=\sqrt{4 \pi n_{j} e^{2} / m_{a}}$ and $\Omega_{j}=e B_{0} / m_{j} c$ are the non-relativistic plasma frequency and the gyro-frequency of species $j$, respectively,

$K_{1}=\frac{\kappa^{2}-1 / 4}{\kappa^{2}}, \quad K_{2}=\frac{2 \kappa-1}{2 \kappa}\left(\frac{\kappa+1}{\kappa}\right)^{3 / 2}$,

$\Lambda_{j}=A_{j}-1, \quad \lambda_{j}=\frac{k_{\perp}^{2} \Theta_{\perp, j}^{2}}{2 \Omega_{j}^{2}}, \quad \xi_{j}=\frac{\omega-n \Omega_{j}}{k_{\|} \Theta_{\|, j}}$

and $Z_{K}\left(\xi_{j}\right)$ is the plasma modified dispersion function given by (Summers et al. 1994)

$Z_{\kappa}\left(\xi_{j}\right)=\frac{\pi^{-1 / 2}}{\kappa_{j}^{3 / 2}} \frac{\Gamma\left(\kappa_{j}\right)}{\Gamma\left(\kappa_{j}-1 / 2\right)} \int_{-\infty}^{\infty} \frac{\left(1+x^{2} / \kappa_{j}\right)^{-\kappa_{j}-1}}{x-\xi_{j}} d x, \mathfrak{J}\left(\xi_{j}\right)>0$.

This paper has been typeset from a $\mathrm{T}_{\mathrm{E}} \mathrm{X} / \mathrm{LAT}_{\mathrm{E}} \mathrm{X}$ file prepared by the author. 